\title{
A Nyström Implementation of an Augmented Electric Field Integral Equation for Low Frequency Electrical Analysis
}

\author{
Yuan Xu, R. J. Adams, and S. D. Gedney \\ Electrical \& Computer Engineering, University of Kentucky \\ Lexington, $\mathrm{KY}$
}

\section{Introduction}

The electric field integral equations (EFIE) solved by method of moments (MOM) [1] is widely used in scattering, antenna, and microwave circuit problems. However, it is well known that EFIE will break down when applied to electrically small structures or low frequency problems. A loop-star decomposition for the RWG discretization overcomes this problem and provides a valid solution from zero to microwave frequencies [2]. But the global loop search becomes a obstacle for complicated geometries. The separated potential integral equation (SPIE) augmented with continuity equations $[3,4]$ uses charge as additional unknowns to remove the imbalance of the vector potential and scalar potential in the conventional EFIE. With the enforcement of the current continuity constraint and proper frequency scaling, the augmented electric field integral equation with RWG discretization avoids the low frequency breakdown and provides an accurate representation of the electric fields.

The locally corrected Nyström (LCN) method [5] is an alternative to MOM for discretizing integral equations and is quite efficient in the implementation of highorder moment solutions. In this paper we describe an application of the LCN discretization method to the augmented EFIE.

It is well known that an RWG representation of surface currents is continuous across each interior edge of a given mesh. However, in a Nyström scheme the current is represented in terms of Legendre polynomials in each cell of the mesh and is generally discontinuous across cell edges. This discontinuity in the current gives rise to line charges on the cell boundaries. We show that it is possible to obtain an accurate and stable discretization of the augmented EFIE by incorporating these line charges as additional degrees of freedom in the LCN discretization procedure.

\section{Formulation}

The EFIE for a PEC surface is expressed as,

$-\vec{t} \cdot \vec{E}^{\mathrm{inc}}(\vec{r})=-\vec{t} \cdot\left[-j k_{0} \eta_{0} \int_{S} \vec{J}\left(\vec{r}^{\prime}\right) G\left(\vec{r}, \vec{r}^{\prime}\right) d s^{\prime}-j \frac{\eta_{0}}{k_{0}} \nabla \int_{S} \nabla G\left(\vec{r}, \vec{r}^{\prime}\right) \cdot \vec{J}\left(\vec{r}^{\prime}\right) d s^{\prime}\right]$ 
where $G\left(\vec{r}, \vec{r}^{\prime}\right)$ is the free space Green's function, $S$ is the surface of the PEC, $\vec{J}\left(\vec{r}^{\prime}\right)$ is the unknown surface current density, $\vec{E}^{\text {inc }}(\vec{r})$ is the incident electric field, and $\vec{t}$ is the vector tangential to $S$ at the observation point $\vec{r}$. The surface $S$ is discretized into curvilinear cells or patches. Since the current in terms of Legendre polynomial is continuous within each cell, the integrals in (1) can be split into integrals over cells. Appling the divergence theorem to the integral over each cell we obtain,

$$
\begin{aligned}
-\vec{t} \cdot \vec{E}^{\text {inc }}(\vec{r})=\left(-\eta_{0}\right)[ & {\left[\vec{t} \cdot j k_{0} \sum \int_{S_{c}} \vec{J}\left(\vec{r}^{\prime}\right) G\left(\vec{r}, \vec{r}^{\prime}\right) d s^{\prime}-\frac{j}{k_{0}} \sum \int_{S_{c}}\left(\vec{t} \cdot \nabla G\left(\vec{r}, \vec{r}^{\prime}\right)\right) \nabla_{\|} \cdot \vec{J}\left(\vec{r}^{\prime}\right) d s^{\prime}\right.} \\
& \left.-\frac{j}{k_{0}} \sum \int_{\partial S_{c}} \hat{e}^{\prime} \cdot \vec{J}\left(\vec{r}^{\prime}\right)\left(\vec{t} \cdot \nabla G\left(\vec{r}^{\prime}, \vec{r}^{\prime}\right)\right) d l^{\prime}\right]
\end{aligned}
$$

where $S_{c}$ is the surface of a cell, $\partial S_{c}$ is the contour bounding $S_{c}, \hat{e}^{\prime}$ is the outward vector normal to $\partial S_{c}$ and tangential to $S_{c}$ at the source point $\vec{r}^{\prime}$. The last term is the contribution from the current discontinuities across edges, and it can be rewritten as a summation over all edges in the mesh,

$$
-\frac{j}{k_{0}} \sum \int_{l_{e}}\left(\hat{e}_{1}^{\prime} \cdot \vec{J}\left(\vec{r}^{\prime}\right)+\hat{e}_{2}^{\prime} \cdot \vec{J}\left(\vec{r}^{\prime}\right)\right)\left(\vec{t} \cdot \nabla G\left(\vec{r}, \vec{r}^{\prime}\right)\right) d l^{\prime}
$$

where $l_{e}$ is an edge of mesh. Vectors $\hat{e}_{1}$ and $\hat{e}_{2}$ are the two edge vectors associated with the cells on either side of edge $l_{e}$.

Substituting the continuity equation on the cell surface,

$$
\nabla_{\|} \cdot \vec{J}(\vec{r})=-j \omega q_{s},
$$

and its reduced version on an edge,

$$
-\hat{e}_{1}^{\prime} \cdot \vec{J}\left(\vec{r}^{\prime}\right)-\hat{e}_{2} \cdot \vec{J}\left(\vec{r}^{\prime}\right)=-j \omega q_{l},
$$

into (2) yields

$$
\begin{aligned}
-\vec{t} \cdot \vec{E}^{\mathrm{inc}}(\vec{r})=\left(-\eta_{0}\right)[ & \vec{t} \cdot \sum \int_{S_{c}} j k_{0} \vec{J}\left(\vec{r}^{\prime}\right) G\left(\vec{r}, \vec{r}^{\prime}\right) d s^{\prime}+\sum \int_{S_{c}}\left(\vec{t} \cdot \nabla G\left(\vec{r}^{\prime}, \vec{r}^{\prime}\right)\right) c_{0} q_{s} d s^{\prime}, \\
& +\sum \int_{l_{e}}\left(\vec{t} \cdot \nabla G\left(\vec{r}, \vec{r}^{\prime}\right)\right) c_{0} q_{l} d l^{\prime}
\end{aligned}
$$

where $q_{s}$ is the surface charge, $q_{l}$ is the line charge on edges, and $c_{0}$ is the speed of light. If $j k_{0} \vec{J}\left(\vec{r}^{\prime}\right), c_{0} q_{s}$ and $c_{0} q_{l}$ are used as the unknown current and charge parameters, the EFIE (6) has no imbalance between vector and scalar potentials. It should be also noted that the charge neutrality still holds for (4) and (5). In fact,

$$
-j \omega \sum \int_{S_{c}} c_{0} q_{s} d s^{\prime}-j \omega \sum \int_{l_{e}} c_{0} q_{l} d l^{\prime}=\sum \int_{S_{c}} \nabla_{\|}{ }^{\prime} \cdot \vec{J} d s^{\prime}-\sum \int_{\partial S_{c}} \hat{e}^{\prime} \cdot \vec{J} d l^{\prime}=0 .
$$


Utilizing the Nyström scheme to discretize the equation and proper scaling of unknowns [4], we arrive at the augmented EFIE with continuity equations,

$$
\left[\begin{array}{ccc}
\mathbf{L}_{\mathrm{v}} & \mathbf{L}_{q_{s}} & \mathbf{L}_{q_{l}} \\
\mathbf{D}_{s} & k_{0}^{2} \mathbf{I} & \mathbf{0} \\
\mathbf{D}_{l} & \mathbf{0} & k_{0}^{2} \mathbf{I}
\end{array}\right]\left[\begin{array}{c}
j k_{0} \mathbf{J} \\
c_{0} \mathbf{q}_{s} \\
c_{0} \mathbf{q}_{l}
\end{array}\right]=\left[\begin{array}{c}
\mathbf{V} / \eta \\
\mathbf{0} \\
\mathbf{0}
\end{array}\right] .
$$

The meaning of parameters in (8) is obvious through the comparison of (8) and (6), (4), (5). $\mathbf{D}_{s}$ and $\mathbf{D}_{l}$ are sparse matrices.

\section{Numerical Example}

To evaluate the proposed Nyström discretization of the augmented EFIE, a PEC sphere with radius $0.5 \mathrm{~m}$ is tested. The condition numbers of the augmented EFIE (8) and conventional EFIE are shown in Figure 1. It is observed that the condition number of augmented EFIE is almost constant over the whole spectrum while the condition number of Nyström EFIE increases sharply as the frequency decreases, and breaks down around $10 \mathrm{e}+5 \mathrm{~Hz}$. Figure 2 shows the near electrical field scattering from a PEC sphere with radius $=1.0$ at frequency $=1 \mathrm{~Hz}$ for vertical polarization at distance $1.5 \mathrm{~m}$ when incident in $\hat{z}$ direction. The solution obtained using the augmented EFIE agrees with MIE series solution. Although not indicated here, the magnetic field solution obtained using the augmented EFIE possesses large errors. This issue will be discussed during the presentation.

\section{References}

[1] R. F. Harrington, Time-Harmonic Electromagnetic Fields, New York: McGraw-Hill, 1961.

[2] J. S. Zhao, W. C. Chew, "Integral equation solutions of Maxwell's equations from zeros frequency to microwave frequencies", IEEE trans. Antenna and Propag., vol. 48, No.10, pp.1635-1645, Oct., 2000.

[3] D. Gope, A. R. Ruehli, and V. Jandhyala, "Solving low-frequency EM-CKT problems using the PEEC method," IEEE Trans. Adv. Packag., vol. 30, no. 2, pp. 313-320, May 2007.

[4] Z. Qian, W. C. Chew, "An augmented electric field integral equation for highspeed interconnect analysis", Microw. Opt. Technol. Lett., vol. 50, no. 10, pp. 2658-2662, Oct., 2008.

[5] S. D. Gedney, "On deriving a locally corrected Nystrom scheme from a quadrature sampled moment method", IEEE trans. Antenna and Propag., vol. 51, No.9, pp.2402-2412, Sept., 2003. 


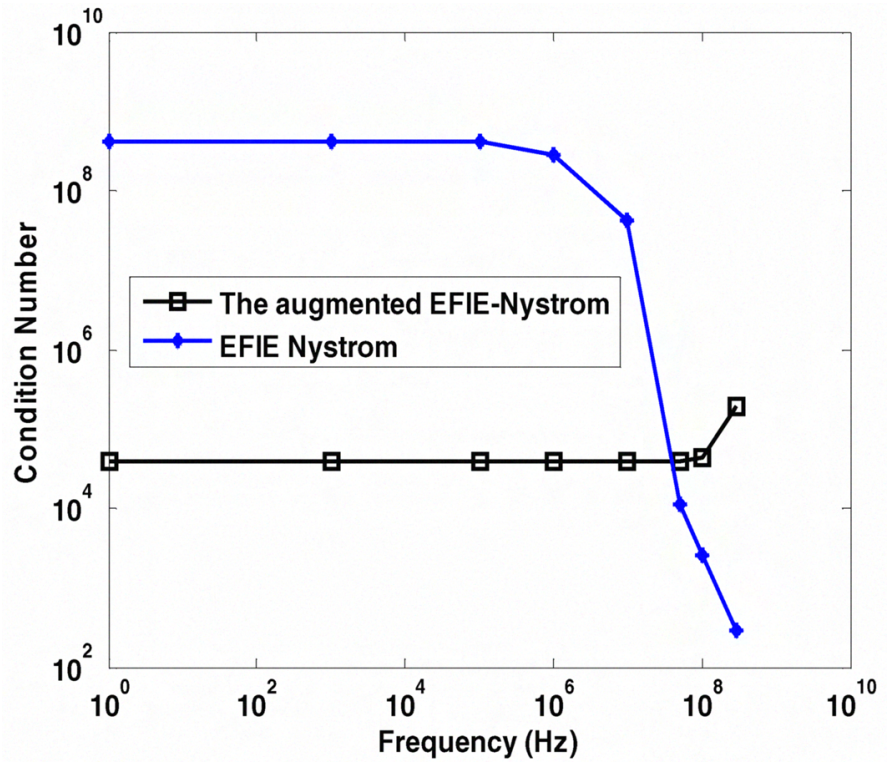

Figure 1. The condition number of Nyström discretization of the augmented and conventional EFIE.

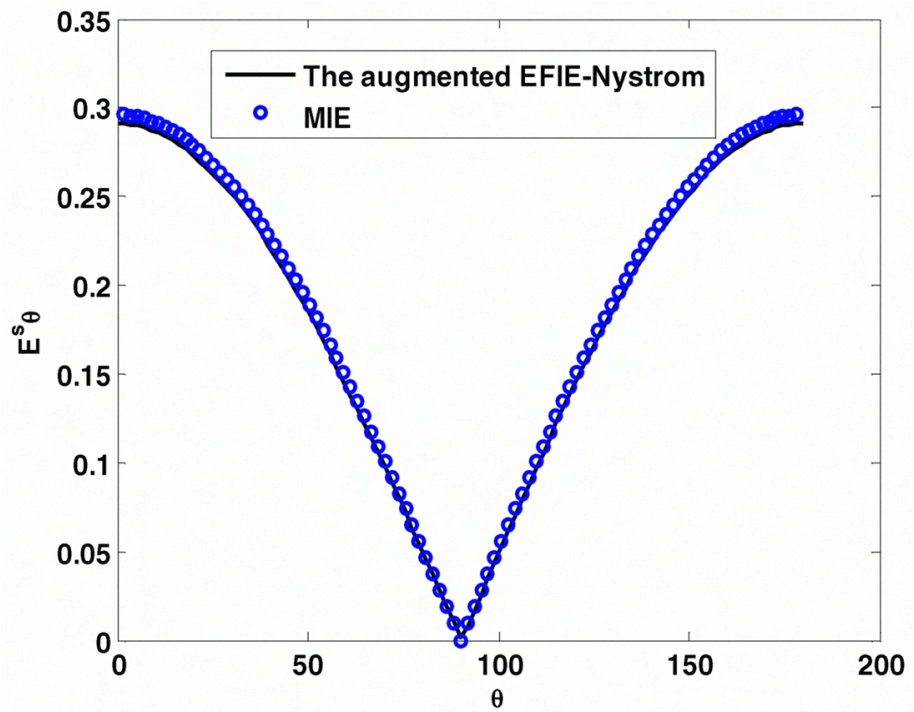

Figure 2. The near electrical field scattering from a PEC sphere with radius $1.0 \mathrm{~m}$ using the augmented EFIE and MIE series. $(\phi=0)$. 